\title{
Synergistic Polaron Formation in the Hubbard-Holstein Model at Small Doping
}

\author{
Alexandru Macridin ${ }^{1}$, Brian Moritz ${ }^{1,2,3}$, M. Jarrell ${ }^{1}$, Thomas Maier ${ }^{4}$ \\ 1 University of Cincinnati, Cincinnati, Ohio, 45221, USA \\ 2 University of Waterloo, Waterloo, Ontario, N2L 3G1, Canada \\ 3 University of North Dakota, Grand Forks, North Dakota, 58202, USA \\ 4 Oak Ridge National Laboratory, Oak Ridge, Tennessee, 37831, USA
}

(Dated: April 17, 2018)

\begin{abstract}
We study the effect of dynamical Holstein phonons on the physics of the Hubbard model at small doping using the dynamical cluster approximation on a $2 \times 2$ cluster. Non-local antiferromagnetic correlations are found to significantly enhance the electron-phonon coupling, resulting in polaron formation for moderate coupling strengths. At finite doping, the electron-phonon coupling is found to strongly enhance the non-local spin correlations, indicating a synergistic interplay between the electron-phonon coupling and antiferromagnetic correlations. Although it enhances the pairing interaction, the electron-phonon coupling is found to decrease the superconducting transition temperature, due to the reduction in the quasiparticle fraction.
\end{abstract}

Introduction. Phonons significantly modify the properties of conventional metals, giving rise to phenomena such as superconductivity and charge-density wave formation. These effects are understood through conventional theories. However, the effect of phonons on strongly correlated metals is less understood. This problem demands investigation given the experimental evidence of significant electron-phonon (EP) interactions in correlated materials such as the cuprates and manganites [1, 2].

Theoretical interest is motivated by the suggestion that electronic correlations greatly enhance the effective EP coupling 3]. In this scenario, the interaction of the holes with their antiferromagnetic (AF) background results in band narrowing which enhances the effective EP coupling, driving the system to the polaron regime even for moderate EP couplings [3, 4, 5]. Quantum Monte Carlo (QMC) calculations [6], for the Hubbard-Holstein (HH) model predict that the EP coupling enhances d-wave pairing in the large coupling regime. Other calculations for the HH or closely related t-J models, including finite size [4, 7, 8, 9] and dynamical mean field approximation (DMFA) calculations 10, 11], suggest that phonons contribute strongly to spin and charge ordering, pairing, and phase separation.

In this letter we employ the dynamical cluster approximation (DCA) 12, 13. to study the two dimensional $\mathrm{HH}$ model at small doping. We investigate the role of the EP coupling on its one and two particle properties. The DOS, charge susceptibility and unscreened local moment indicate band narrowing and polaron formation at moderate EP couplings. Since this implies reduced mobility of the holes, antiferromagnetism is strongly enhanced at finite doping, but not at half filling. We find that the effective AF exchange as well as the pseudogap observed in both the single-particle spectra and the uniform spin susceptibility are nearly independent of the EP coupling. $\mathrm{D}$-wave superconductivity is suppressed by the phonons due to the reduction of quasiparticle weight.
The HH Hamiltonian reads

$$
\begin{aligned}
H & =-t \sum_{\langle i j\rangle \sigma}\left(c_{i \sigma}^{\dagger} c_{j \sigma}+c_{j \sigma}^{\dagger} c_{i \sigma}\right)+U \sum_{i} n_{i \uparrow} n_{i \downarrow} \\
& +\sum_{i} \frac{p_{i}^{2}}{2 M}+\frac{1}{2} M \omega_{0}^{2} x_{i}^{2}+g n_{i} x_{i} .
\end{aligned}
$$

with a nearest-neighbor hopping $t$, an on-site Coulomb repulsion $U$, dispersionless optical phonons with frequency $\omega_{0}$ and an on-site EP coupling $g$. The dimensionless EP coupling is defined as $\lambda=2 g^{2} /\left(2 M \omega_{0}^{2} W\right)$ and represents the ratio of the single-electron lattice deformation energy $E_{p}=g^{2} /\left(2 M \omega_{0}^{2}\right)$ to half of the electronic bandwidth $W / 2=4 t$. We present results for small phonon frequency $\omega_{0}=0.3 t, U=8 t$ and values of $\lambda$ in the weak and intermediate coupling regime, $\lambda \leq 0.7$.

To study the Hamiltonian (11) we employ the DCA, a cluster mean-field theory which maps the original lattice model onto a periodic cluster of size $N_{c}=L_{c}^{2}$ embedded in a self-consistent host. Correlations up to a range $L_{c}$ are treated explicitly, while those at longer length scales are described at the mean-field level. With increasing cluster size, the DCA systematically interpolates between the single-site DMFA result and the exact result, while remaining in the thermodynamic limit. Cluster mean field calculations on the simple Hubbard model are found to reproduce many of the features of the cuprates, including a Mott gap and strong AF correlations, d-wave superconductivity and pseudogap behavior [13].

We solve the cluster problem using a quantum Monte Carlo (QMC) algorithm [14] modified to perform the sum over both the discrete field used to decouple the Hubbard repulsion, as well as the phonon field $x$. The space of configurations of the latter field is significantly larger than the former, so the $\mathrm{HH}$ code requires significantly more CPU time than required for the Hubbard model. Thus, present calculations are restricted to clusters of size $N_{c}=4$. The Maximum Entropy method [15] is employed to calculate the real frequency spectra. 


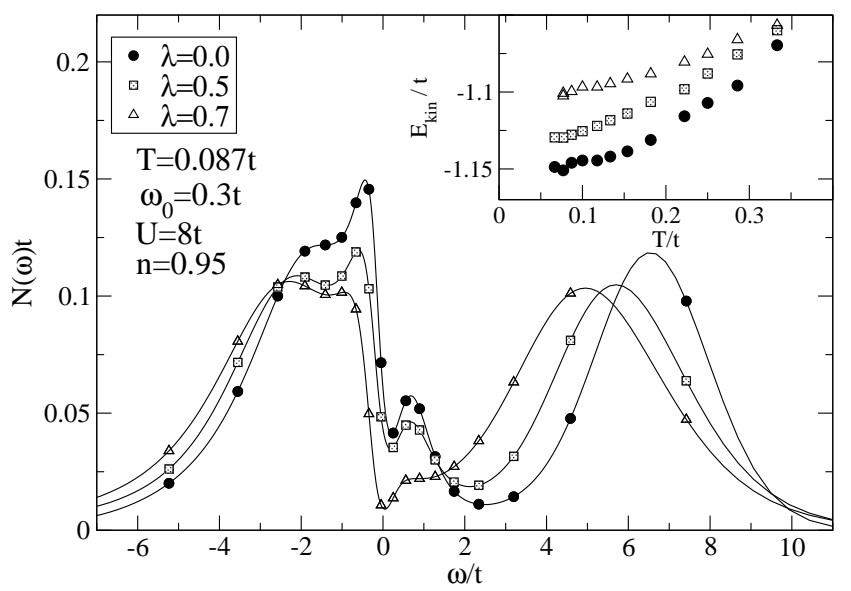

FIG. 1: Single-particle DOS for several values of $\lambda$. The pseudogap width is negligibly dependent on $\lambda$. Inset: the electronic kinetic energy, $E_{k i n}=\sum_{k \sigma} \epsilon(k) n_{k, \sigma}$, versus T. $N(0)$ and the kinetic energy gain are suppressed with increasing $\lambda$.

Results. The electronic DOS at 5\% doping is plotted in Fig. 1] With increasing $\lambda$ the DOS at the Fermi energy, $N(0)$, is strongly suppressed. The pseudogap in the DOS, as measured by the peak to peak distance, does not depend much on $\lambda$. However, the peaks adjacent to the pseudogap are strongly suppressed by the EP coupling. The EP coupling also reduces the separation of the Mott peaks which is a measure of $U_{\text {eff }}$. The reduction depends on the phonon frequency (not shown) and for small $\omega_{0}$ we find that it is much smaller than that expected in the antiadiabatic limit $\left(U_{\text {eff }}=U-\lambda W\right.$ when $\left.\omega_{0} \rightarrow \infty\right)$.

The inset in Fig. 1]shows that the electronic kinetic energy gain at low temperatures decreases with increasing $\lambda$. The quasiparticle fraction, which will be discussed below (see Fig. 4), also falls with increasing $\lambda$. These results, together with the suppression of $N(0)$ and the peaks adjacent to the pseudogap, which in previous calculations were associated with quasiparticles [13], are consistent with a significant decrease in the effective electronic bandwidth and a reduction of the coherent part of the electronic Green's function due to EP coupling.

The local dynamical charge susceptibility is plotted in Fig. 2(a). It develops a narrow peak with increasing $\lambda$, suggesting the development of a nearly localized band of polaron charge carriers. When dynamical phonons are considered there is no self-localization, making it difficult to define a critical $\lambda_{c}$ for polaron formation. For $\lambda=0$ the width of the peak is of order of $t$, while for $\lambda \geq 0.5$ the width of the peak becomes smaller than the phonon frequency $\omega_{0}$ which we take to indicate the crossover to a polaron regime. Note that the peak found in the DCA (solid lines) is much narrower than that found by the DMFA (dashed lines) for the same parameters [16]. This is consistent with the argument [3] that the effective EP coupling is enhanced by non-local AF correlations, which

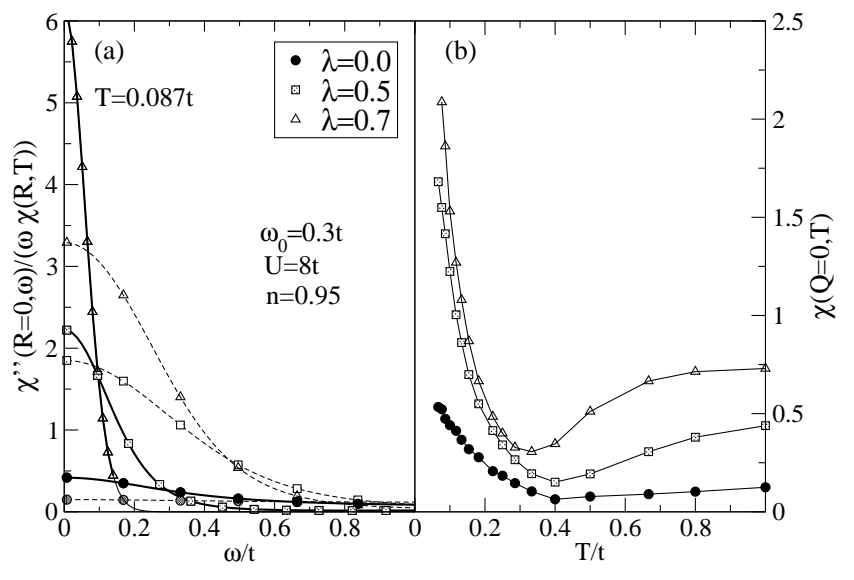

FIG. 2: (a) The local dynamic charge susceptibility for several values of $\lambda$. The dashed (solid) lines are DMFA (DCA) results. A narrow band of low energy charge excitations develops as $\lambda$ increases which indicates polaron formation. (b) Static charge susceptibility (compressibility) versus $T$ for the same values of $\lambda$. The strong enhancement indicates the tendency to phase separation.

are present in the DCA but not in the DMFA. Polaron formation is also associated with phase separation, since the electronic band is narrowed, i.e. $t_{\text {eff }}$ is reduced, and the effective AF exchange $J_{e f f}$, as we will show below, essentially remains unchanged for small $\omega_{0} / t$. An increased $J_{\text {eff }} / t_{\text {eff }}$ leads to phase separation since the system can gain more energy from spin exchange than it can from the kinetic energy of the carriers [17]. In accord with this argument, the bulk charge susceptibility (or compressibility), shown in Fig. 2(b), is strongly enhanced by the EP coupling (for larger values of $\lambda$ than those shown, it diverges at low $T$ ) indicating a charge ordering transition (phase separation).

The dynamic cluster spin susceptibility is plotted in Fig. [3 (a) for $Q=(0, \pi)$. Due to remnant AF spin correlations, it has a magnon peak at an energy proportional to the effective exchange interaction $J_{\text {eff }}$. The fact that $J_{\text {eff }}$ increases very slowly with $\lambda$ is expected from perturbation theory in $t$ [18 and exact diagonalization results [19] when $\omega_{0}$ is small. $J_{\text {eff }}$ is independent of $\lambda$ when $\omega_{0} \rightarrow 0$ since the lattice cannot respond during the virtual hopping between near neighbors which lowers the energy of the singlet relative to the triplet.

The unscreened moment $\mu^{2}=\left\langle\left(n_{\uparrow}-n_{\downarrow}\right)^{2}\right\rangle$ is plotted in Fig. 3(b). In the DCA calculation, increasing $\lambda$ greatly increases the temperature dependence of $\mu^{2}$, but at low temperatures, $\mu^{2}$ is essentially independent of $\lambda$. In the DMFA calculation, at the same temperatures the EP coupling is found to significantly reduce $\mu^{2}$ due to the tendency of the phonons to reduce the on-site correlations 10]. In the DCA, the reduction of the effective hopping amplitude due to the larger effective EP coupling (i.e. enhanced polaron formation) counteracts 


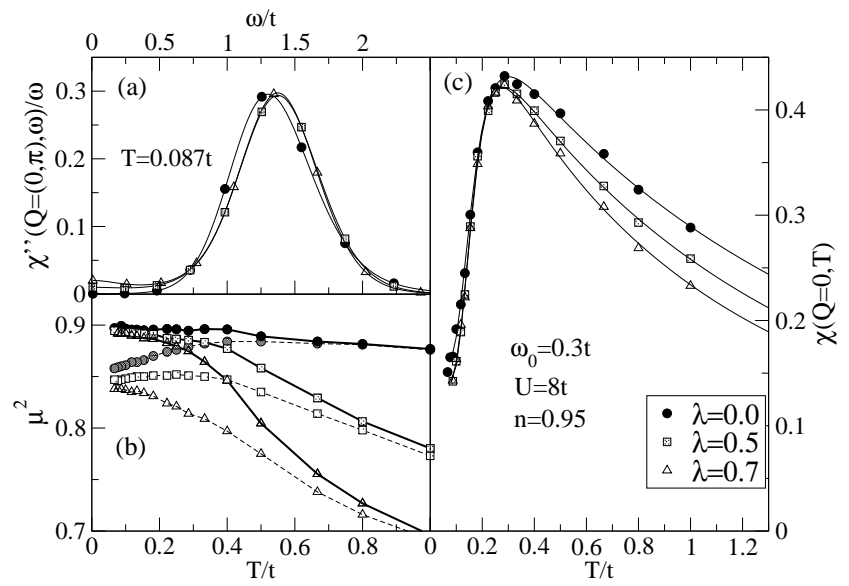

FIG. 3: (a) The dynamic cluster spin susceptibility with $Q=$ $(\pi, 0)$. The location of the peak is a measure of the effective spin exchange $J_{\text {eff }}$. (b) The unscreened moment $\mu^{2}=\left\langle\left(n_{\uparrow}-\right.\right.$ $\left.\left.n_{\downarrow}\right)^{2}\right\rangle$ versus $T$, calculated with the DCA (solid lines) and the DMFA (dashed lines). The EP coupling strongly increases the temperature-dependence of $\mu^{2}$ but the low-temperature value is unaffected. (c) The bulk spin susceptibility versus T.

the reduction of on-site correlations, thus leaving the low temperature unscreened moment unaffected.

The bulk spin susceptibility is plotted in Fig. 3(c). Unlike the charge susceptibility, the low temperature bulk spin susceptibility is essentially independent of $\lambda$. When the bulk susceptibility is divided by the unscreened moment $\mu^{2}$, the four curves collapse upon each other (not shown), with small deviations consistent with the slight increase of $J_{\text {eff }}$ with $\lambda$. This indicates that the temperature and $\lambda$ dependence of the bulk susceptibility, is due predominantly to its effect on the unscreened moment. The emergence of the pseudogap is associated with the suppression of the spin excitations and it is interesting to notice the weak $\lambda$-dependence of both the low-T bulk spin susceptibility and the pseudogap in the DOS. This suggests that the pseudogap energy scale is $J_{\text {eff }}$.

The AF transition temperature $T_{N}$ at half filling $(n=$ 1 ) is weakly dependent on $\lambda$ as shown in Fig. 4(a). It increases only slightly, consistent with the increase in $J_{\text {eff }}$ with $\lambda$ found in Fig. 3(a). However, when the system is doped, $T_{N}$ increases dramatically with $\lambda$ since polaron formation suppresses the mobility of the holes that disrupts the AF order. Hence, not only do the AF correlations enhance the EP coupling [3] and polaron formation [4], but the EP coupling also strongly enhances antiferromagnetism in the doped model. This establishes a synergistic cooperation between the EP coupling and AF correlations. Note that at half filling and small dopings, unlike DCA, the DMFA predicts a reduction of $T_{N}$ associated with the decrease in $U_{\text {eff }}$. However, like the DCA, at larger doping the AF order is enhanced in DMFA due to the decrease of charge carrier mobility, although the

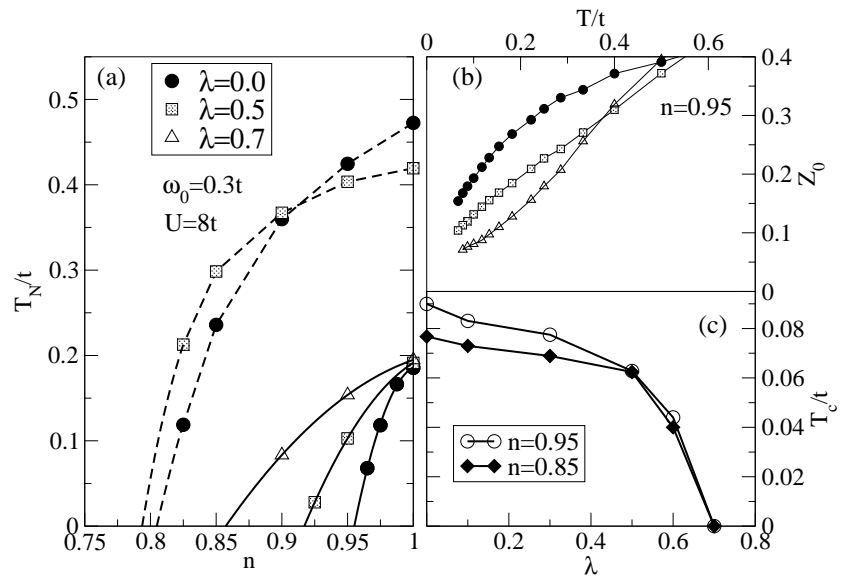

FIG. 4: (a) $T_{N}$ versus filling for several values of $\lambda$ calculated with the DCA (solid lines) and the DMFA (dashed lines). When $n=1$, the DCA $T_{N}$ increases slightly, consistent with a small increase in $J_{\text {eff }}$ shown in Fig. 31 a). At small doping, $T_{N}$ is enhanced by the EP coupling in contrast to the DMFA result where $T_{N}$ is suppressed. (b) The Matsubara quasiparticle fraction $Z_{0}=1 /(1-\operatorname{Im} \Sigma(K, \pi T) / \pi T)$ versus $T$ for several values of $\lambda$. (c) Superconducting transition temperature versus $\lambda$ at $5 \%$ and $15 \%$ doping.

effect is not as pronounced.

It is unclear a priori if the EP coupling will enhance superconductivity. For $U>6 t$ the EP vertex at small momentum transfers increases with increasing $U$, hence contributing to a $\mathrm{d}$-wave pairing interaction [6]. However, the EP coupling also suppresses $N(0)$ as well as the Matsubara quasiparticle fraction $Z_{0}=1 /(1-\operatorname{Im} \Sigma(K, \pi T) / \pi T)$. It is plotted in Fig. प(b) for different $\lambda$ for $K=(0, \pi)$. As $T \rightarrow 0, Z_{0} \rightarrow Z$ where $Z$ is the quasiparticle fraction defined by the derivative of the real-frequency self energy. At low temperatures the quasiparticle fraction is strongly reduced for large $\lambda$. The superconducting transition temperature is plotted in Fig. 目(c) versus $\lambda$ at $5 \%$ and $15 \%$ doping. The leading pairing instability remains even-frequency d-wave. $T_{c}$ falls with increasing $\lambda$, indicating that the reduction of $N(0)$ and the quasiparticle fraction outweighs the enhanced interaction. Alternatively, several authors have proposed that pairing in the cuprates could be driven by kinetic energy gain 20, 21]. The suppression of the kinetic energy seen in Fig. 1 is consistent with the suppression of $T_{c}$ in such scenarios.

While our results are valid at small $\omega_{0}$, qualitative differences are expected when it increases. However, note that in real materials the phonon energy is even smaller than our value, e.g. in cuprates $\omega_{0} \approx 0.10 t, 0.15 t[1]$.

Several experimental features in the cuprates can be addressed with our results. The enhancement of the EP coupling due to AF correlations can explain the large $\lambda \approx 1.5$ seen in the photoemission spectra in the un- 
derdoped cuprates [1], whereas density functional calculations predict a moderate EP coupling $\lambda \approx 0.4[22$. The enhancement of the charge susceptibility (Fig. 2) shows that EP coupling is relevant for the charge inhomogeneities observed in many high $T_{c}$ materials 23].

There is evidence in the literature that AF correlations can enhance EP coupling, and mixed results regarding polaron formation and pairing. Exact diagonalization calculations with both adiabatic [4] and dynamic [8, 9] phonons, finite-size QMC [6] and diagrammatic QMC [ 5 all suggest that AF correlations strongly enhance the effect of EP coupling. Those with dynamic [9] phonons find evidence that the phonons can enhance the equal time AF correlations. However, these calculations have an extremely truncated phonon Hilbert space that limits their ability to properly describe polaron formation. Perhaps as a consequence, these calculations suggest that Holstein phonons enhance d-wave superconductivity. Bipolaron calculations [18, 24] suggest enhanced formation of mobile intersite singlets for intermediate EP coupling. However, the weak $\lambda$-dependence of the spin susceptibility, Fig. 3 (c), excludes this scenario. Bipolaron calculations, like exact diagonalization at small doping, are restricted to one or two carriers, while the DCA addresses finite doping in the thermodynamic limit. In recent weakcoupling functional RG calculations, Holstein phonons were found to reduce the AF susceptibility, as well as pairing (but certain lower symmetry modes enhance pairing) 25]. However, these results neglect polaronic effects.

Conclusion. We study the effect of Holstein phonons on the properties of the two-dimensional Hubbard model using the DCA and QMC. At small doping we find that $N(0)$ and the quasiparticle fraction are strongly suppressed with increasing EP coupling. This, together with a strongly increased charge susceptibility and the formation of a narrow band of charge excitations in the dynamic susceptibility, are taken as strong evidence for the formation of polarons. The evidence is far stronger in the DCA than the DMFA results, consistent with the argument that non-local spin correlations strongly enhance the EP coupling [3]. The spin properties captured by the DCA show that while the AF exchange $J_{\text {eff }}$ increases slightly, the primary effect of the EP coupling is to increases the temperature dependence of the unscreened moment $\mu^{2}$ while essentially preserving its low-temperature value. Despite the reduction of $U_{\text {eff }}$ the phonons do not suppress the low- $T$ moment, since the polarons suppress the hybridization of the sites with their environment. The AF transition temperature $T_{N}$ at half filling increases slightly, by an amount commensurate with the increase in $J_{\text {eff }}$. However, at finite doping $T_{N}$ increases dramatically, due to the suppression of the itinerant holes mobility by polaron formation. Thus not only do the AF correlations increase the effective EP coupling but also the EP interaction increases the AF correlations. We find that both the magnitude of the pseudogap in the single particle DOS and the pseudogap signature in the bulk spin susceptibility are not affected significantly by EP coupling, suggesting that the pseudogap energy scale is $J_{\text {eff }}$. Finally, although the EP coupling is expected to increase the d-wave pairing interaction [6], we find that the superconducting $T_{c}$ is actually suppressed due to the reduction of the quasiparticle fraction $Z$ and $N(0)$. However it is possible that couplings to other phonon modes with lower symmetry, may contribute an interaction which will enhance the d-wave $T_{c}$. This will be a subject of future studies.

We thank G. Sawatzky, T. Devereaux, C. Honerkamp, R. Scalettar and H.-B. Schüttler for useful discussions. This research was supported by NSF DMR-0312680, CMSN DOE DE-FG02-04ER46129, ONR N00014-05-10127 and NSF SCI-9619020 through resources provided by the San Diego Supercomputer Center. TM acknowledges the Center for Nanophase Materials Sciences, sponsored by the Division of Scientific User Facilities, U.S. Department of Energy. BM acknowledges the UND Computational Research Center, supported by NSF EPS0132289 and EPS-0447679, and the hospitality of the Pacific Institute of Theoretical Physics.

[1] A. Lanzara et al., Nature (London) 412, 510 (2001); T. Cuk et al., Phys. Stat. Sol. (b) 242, 11 (2005).

[2] A. J. Millis et al., Phys. Rev. Lett. 74, 5144 (1995); J. M. De Teresa et al., Nature (London) 386, 256 (1997); N. Mannella et al., Nature (London) 438, 474 (2005).

[3] J. Zhong et al., Phys. Rev. Lett. 69, 1600 (1992).

[4] P. Prelovšek et al., Phys. Rev. Lett. 96, 086402 (2006).

[5] A. Mishchenko et al., Phys. Rev. Lett. 93, 36402 (2004).

[6] Z.B. Huang et al., Phys. Rev. B 68, 220507(R) (2003).

[7] B. J. Alder et al., Phys. Rev. Lett. 79, 3022 (1997).

[8] J. Riera and A. Moreo, Phys. Rev. B 73, 014518 (2006).

[9] T. Sakai et al., Phys. Rev. B 55, 8445 (1997).

[10] G. Sangiovanni et al., Phys. Rev. Lett. 94, 026401 (2005).

[11] M. Capone et al. Phys. Rev. Lett. 92, 106401 (2004).

[12] M. H. Hettler et al., Phys. Rev. B 58, R7475 (1998); M. H. Hettler et al., Phys. Rev. B 61,12739 (2000).

[13] Th. Maier et al., Rev. Mod. Phys. 77, 1027 (2005).

[14] M. Jarrell et al., Phys. Rev. B 64, 195130 (2001).

[15] M. Jarrell et al., Physics Reports 269 No.3, 133 (1996).

[16] At low $T$ the DMFA DOS and dynamic charge susceptibility develop phonon features at $\omega \approx \omega_{0}$ not found with the DCA at the temperatures studied.

[17] V. J. Emery et al., Phys. Rev. Lett. 64, 475 (1990).

[18] A. Macridin et al., Phys. Rev. B 69, 245111 (2004).

[19] W. Stephan et al., Physics Lett. A 227, 120-126 (1997).

[20] Th. Maier et al., Phys. Rev. Lett. 92, 027005 (2004).

[21] E. Arrigoni et al., Phys. Rev. B 69, 214519 (2004); A. Bianconi et al., J. Phys.: Cond. Matter 12, 10655 (2000).

[22] O. Andersen et al., J. Low Temp. Phys. 105, 285 (1996).

[23] J. M. Tranquada et al., Nature (London) 375, 561 (1995); J. E. Hoffman et al., Science 295, 466 (2002).

[24] A. La Magna et al., Phys. Rev. B 55, 14886 (1997).

[25] C. Honerkamp et al., preprint, cond-mat/0605161 